\title{
Developing a Web-based Environment in Supporting Students Team-working and Learning in a Problem-based Learning Approach
}

\author{
Hsiu-Ping Yueh \\ Department of Agricultural Extension, \\ National Taiwan University \\ Academic Center for Computing and Media \\ Studies, Kyoto University \\ yueh@mm.media.kyoto-u.ac.jp
}

\begin{abstract}
This paper presents a case study of applying problem-based learning in the format of web-based environment. It first describes the features and rationale behind problem-based learning approach, and then introduces the development of web-based environment as well as its implementation of a university classroom in facilitating students' teamworking. It then discusses results of the evaluation conducted to understand the effectiveness of this technology application. Findings show that most students have positive attitude toward this application, and they think the system can help their team-working in the problem-based learning process. Also students' feedbacks demonstrate that their learning from the web-based problem-based learning approach is significantly improved.
\end{abstract}

\section{Introduction}

The Problem-based Learning (PBL) was launched as an alternative approach to traditional professional education and has been wide spread all over the world either in professional education programs or general higher education practices. Besides, in the past decade, scholars globally are devoted themselves to applying new technology into instructional activities and also are interested in studying the effectiveness of teaching and learning with technology. This study adopts the problem-based learning approach and implements it in a university agricultural information course with technology infusion to facilitate the process of students' team-working and learning. And this paper describes the development and application of a webbased environment designed to facilitate the process of problem-based learning, which provides a detailed records of all the steps followed during the process in

\author{
Wei-Jane Lin \\ Department of Agricultural Extension, \\ National Taiwan University \\ minniewjlin@ntu.edu.tw
}

an attempt to analyze how student reason and learn throughout the problem-based learning sessions.

\section{Rationale of problem-based learning}

All printed material, including text, illustrations, and charts, must be kept within a print area of $6-1 / 2$ inches $(16.51 \mathrm{~cm})$ wide by $8-7 / 8$ inches $(22.51 \mathrm{~cm})$ high. Do not write or print anything outside the print area. All text must be in a two-column format. Columns are to be $3-1 / 16$ inches $(7.85 \mathrm{~cm})$ wide, with a $3 / 8$ inch $(0.81 \mathrm{~cm})$ space between them. Text must be fully justified.

Problem-based learning was characterized as a collection of carefully crafted, ill-structured problems that reveal the underlying principles and concepts of a knowledge domain through descriptions of real life events and experiences, and serve as the stimulus and focus for student activity [7]. It was originally defined as a student-centered instructional method, which puts students in a real world situation and expects them to find out the problems they are interested. Students discover the problems by themselves, trying to figure out their own solution to the problem and generating new learning needs through the process of problem inquiring [1]. Comparing to the traditional instruction method, researches also show that students perform different ways of knowledge generating and processing in problem-based learning [2].

The widespread use of the PBL in medical schools around the world has been accomplished by a large number of research studies on PBL process and outcomes. PBL is introduced to medical education in Taiwan since 1992 [6], most of related researches and experiments are directed by medical schools, too. But except for medical education, problem-based learning can be also applied to many kinds of curricula such as business, law, education, engineer, science and ethics, etc [10]. The adequacy of application largely depends 
on the characteristics of the knowledge domain, which directs the complexity of the problems in problembased learning.

Broadly, two main areas of research on PBL can be identified: (a) comparison with conventional curricula regarding acquired knowledge, reasoning skills, motivation and attitudes towards learning; (b) the evaluation of students' satisfaction with and perspectives on learning using the method [3]; and (c) roles such as instructors, tutors and students play in the process of problem-based learning session. Growing concern about problem-based learning modules (PBLMs) and problem-based learning systems (PBLS) are also expressed in many papers, in which some possible methods and mechanisms of improving PBL were suggested.

However, most of these systems concern the process of problem-based learning, few attention is paid to the content and learning students generated in the process. This may be partly due to the extreme diversity of the learning environments created during PBL sessions, which cannot easily be captured by the tutor or even by a professional observer. In order to overcome this difficulty, this study intends to apply web forum companied with the instructional design of the course website, to effectively provide and record all the information in the learning environment and also attempt to facilitate the PBL process student involved.

\section{Technology in PBL approach}

With the rapid development of Internet, web-based environment performs many kinds of information formats and functions exceed which conventional classrooms could provide. The vantage of world-wideweb includes:

(a) Multiple presentation via medium in the same time.

(b) Non-linear connection allow browsers/learners to select information they are interested.

(c) Comment protocol to contain and integrate many kinds of media types.

(d) Easy to extend additional, interactive functions into one website [4].

Learning through web is also convenient for students to interact and share with information, the same teaching material can be presented to the students, assuring uniformity of criteria used to access the information, each working group and individual can evolve their own strategy of moving through a problem, and the records of the students' work can be easily recovered and analyzed [8].

Although there are more and more attentions drawn by instructors to explore the possibility of integrating technology or web-based environment in classroom, and maybe in the problem-based learning approach, however, few of them are systems that truly integrate this PBL approach in the technology or web systems. Some may use a web discussion board or forum to facilitate students' discussion; others may use a course or learning management system to put instructional materials online, yet they can just support part of the learning that students may need in their team-working or learning process of PBL approach.

\section{Design of web-based PBL environment}

This study tries to develop a prototype of web-based PBL learning environment which contains components of general course management system as well as special features of problem-based learning support. Throughout the use of discussion forums and group PBL working system developed for each group to help group members share their experience and thoughts online, it also help the group to automatically collect their team-working data and products which can also be aggregated as group portfolios to present their team work products in the same web-based environment.

\subsection{Course background and needs analysis}

This study was conducted in the context of a course for both graduate and undergraduate levels entitled of "Telecommunication Systems for Agricultural Information" offered by the Communication Program of a university in northern Taiwan. The main course objective is to improve the students' understanding of the effective application of communication process and technology available to facilitate the diffusion of innovative technologies and communication strategies of agricultural information. In this context it was thought to be relevant with implementation of the PBL methodology as an instructional and learning strategy with the following consideration. Those are to develop the abilities of:

(a) Analysis and Inference: students are expected to define the problems of the communication of agricultural information in the real world, and to explore the causes and solutions to the problems.

(b) Data collection and management: students need to learn to collect and analyze whatever resources that might be related to the problems they defined with theories and practical cases.

(c) Expertise and mastery of the domain knowledge: students are asked to discuss in their group and provide any information they referred on the course website. By doing so, all information would be recorded and used for their project works so that they can further analyze the information and to come up with their 
solutions. Moreover, it is hoped throughout the process, students in a group can also construct their knowledge together as many researchers argued that the integration of technology in learning should be able to enhance students' knowledge construction [5], especially for collaborative learning.

4.2.1. Overviews of the system. As mentioned in previous session, this system contains of functions of general content management system such as announcement, course information, lecture notes of each class, and instructor's contacts (see Figure1).
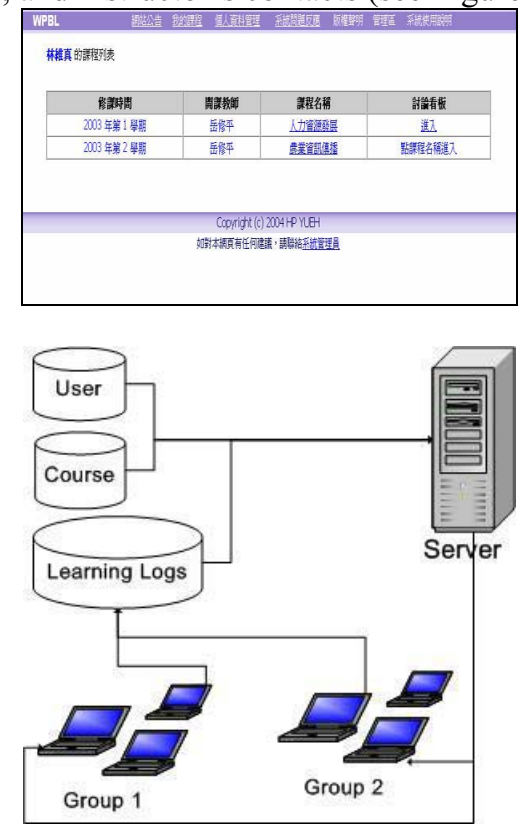

Figure1: The course website

4.2.2. Functions for PBL team-working. There are some functions developed specifically for students' team-working in the problem-based learning process such as the introduction to the PBL approach (Figure 2 ); the discussion forums that include different phases of PBL processes (Figure 3); the PBL working system for collaborative work including all members contribution (Figure 4); and the group portfolios as final products of team-working (Figure 5).

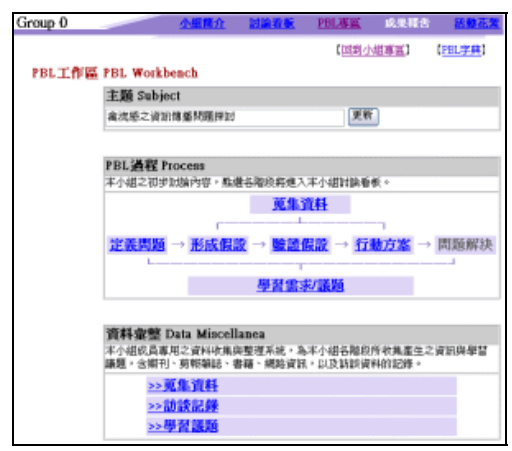

Figure2: Introduction to PBL

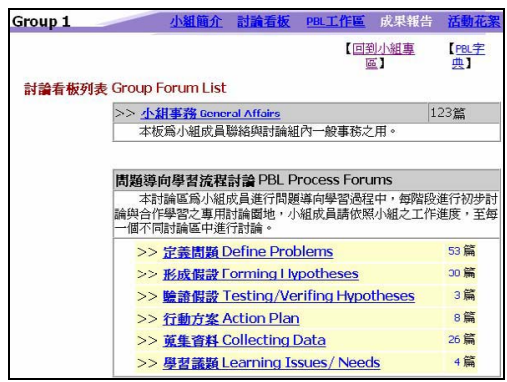

Figure3: Group discussion forums
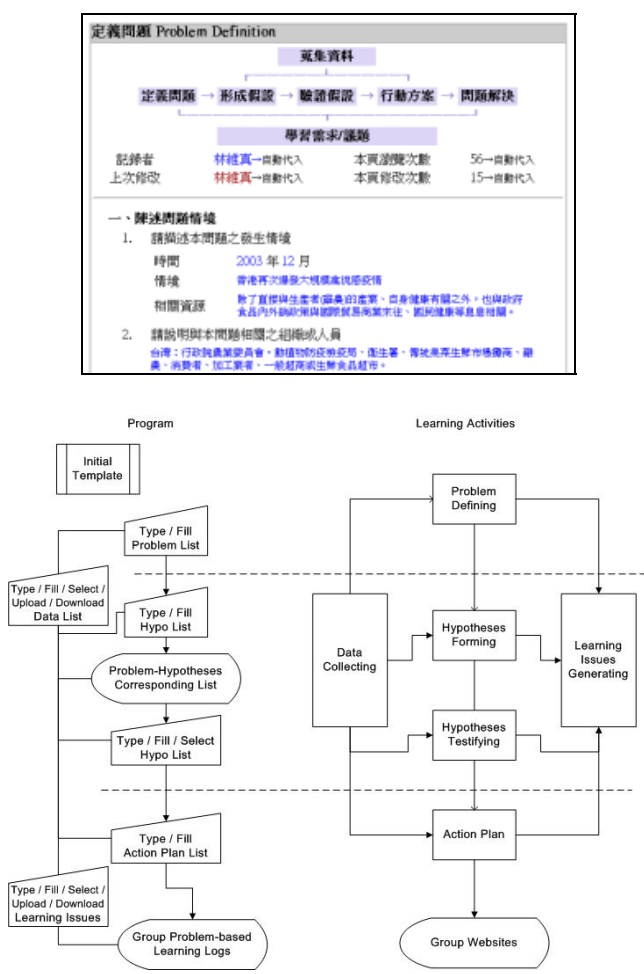

Figure4: PBL working system 


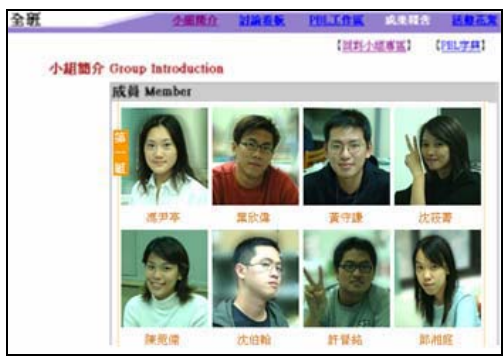

Figure5: PBL group portfolios

\section{Evaluation of the system}

The researchers try to investigate how the webbased environment we developed can facilitate students' learning in the problem-based learning approach and conduct an evaluation study in collecting students' attitudes toward this web-based PBL system. A class of 18 students divided into 3 groups registered the course and participated in the study in spring 2004. The instructor introduces the process on the course website as: define problem; form hypotheses; collect data; verify hypotheses; propose solutions with an action plan to ensure the group working process. At the end of the semester, questionnaires with likert type items and open-ended reflections are designed by the researchers and distributed to students to determine the effectiveness of the system with PBL approach.

\section{Results of the study}

Based on results of the questionnaires, most students showed very positive attitude toward the webbased learning environment that include all those online course information as well as many functions and mechanisms the website provided in support of their group team-working in problem-based learning. Some students who play a very active roles in their groups by sharing a variety of information they collected and posting a lot of comments on the system. They also lead the discussions and inspire other members to participate more actively and this kind of enthusiasm truly improves the group performance and helps their team work online. This is especially valued in the groups that are composed of students from different departments because they can not see each other often in person but still can effectively work together online.

All groups of students comment that they thought the PBL working system that is embedded in the course system is very helpful to their work throughout PBL process. By following the problem-based learning process with scaffolding of the PBL working system, it makes their reasoning of the problem more logistically and effectively. Furthermore, design of the system also makes it easier for them to look up and integrate all information and resources needed that might collected or contributed by different group members at various time points. Furthermore, with clear records of all information of each phases of PBL process, the instructor and tutors can easily give feedbacks to students in a proper way, which also receive students' positive appraises by their reflections.

Through the instructor and tutors' evaluation, the problems all groups of students chose and their working processes are appropriate and closely related to the core issues of agricultural information. Besides, findings suggest that in order to form the hypotheses and propose the solution with an action plan, students need to make connections among all relevant sources of information that they learned before to figure out the possible ways, most students report that this is the first time they know what and why they learn rather than just memorizing the content on the textbooks, echoing findings by Schmidt [9], problem-based learning facilitate the process of learning via activating students' prior knowledge.

\section{Conclusion}

Problem-based learning approach encourages instructors to uses authentic problems in real life as stimulus and focus for student activity in the context of teamwork. Students need to collect a variety of information and engaged in the group discussions to successfully complete their PBL work. Web-based environment with specific PBL working functions can effectively facilitate students' needs of information collection and sharing, as well as their discussions. It also helps record their group learning processes and can scaffold their works in the PBL process. This study proves a good design and development of a webbased PBL system can successful support students' effective team-working and learning in the PBL approach.

Results of the study also show that students of the class all view the PBL approach as well as the webbased environment as an innovation in their learning experiences. Although at the beginning of the class, some students might feel confused with this PBL pedagogy due to the requirement of highly demand of collaborative working with group members as well as the self-directed learning approach. However, along with the progress the group has made, with clarification in class meetings and online tutoring by instructor and tutors, students find it's very valuable to have this kind of learning experience especially they can finally come up with constructive and valuable 
solutions to the problem and even can propose and will be accepted by the stakeholders of the problem.

As the researchers' reflection, this can not be done successfully without a proper design and technology implementation of the web-based PBL system. The important information recorded by the web-based system could show how students in the group interact with each other to confront, identify, testify and solve a problem. It not only can provide valuable information to instructors in the class to understanding more about students learning and to help them with better tutoring in the process, but also which can provide empirical evidence for researchers of problem-based learning as well as professionals in computing technology in education in the future.

In summary, this study highlights the importance of matching the curriculum goal with appropriate selection and adoption of information technologies or web systems as well as instructional pedagogies to maximize the impact of technology in education.

\section{References}

[1] Barrows, H., \& Tamblyn, R., Problem-based learning, Springer, New York, 1980.

[2] Barrows, H., Practice-based learning: Problembased learning applied to medical education. Springfield, Illinois, 1994.

[3] Caplow, J.A., Donaldson, J.F., Kardash, C.A. \& Hosokawa, M., "Learning in a problem-based medical curriculum: students' conceptions", Medical Education, 31, 1997, pp. 440-447.
[4] Connick, P.G., "Issues and trends to take us into the 21 century", In T.E.Cyrs (Ed.), Teaching and learning at a distance: What it takes to effectively design, deliver and evaluate programs, Jossey-Bass, Inc., California, 1997. pp. 7-12.

[5] Jonassen, D.H., Peck, K., and Wilson, B.F., Learning with technology. A constructivist approach, Prentice Hall, Upper Saddle River, NJ, U.S.A., 1999.

[6] Lue, B.H., Leung, K.K., Lee, M.B., Lee, Y.J., \& Hsieh, B.S. "Evaluation of the courses using smallgroup tutorial", Journal of Medicine Education, 2(4), 1998, pp. 386-396.

[7] Norman, G., \& Schmidt, H., "The psychological bases of problem-based learning: A review of the evidence", Academic Medicine, 67(9), 1992, pp.557565.

[8] Prentice, J.W. \& Kenny, G.N., "Microcomputers in medical education", Medical Teacher, 8, 1996, pp. 918.

[9] Schmidt, H.G., "Problem-based learning: rationale and description", Medical Education, 17, 1983, pp. 11-16.

[10] Smit, P.B.A., Verbeek, J.H.A.M., \& de Buisonje, C.D., "Problem based learning in continuing medical education: a review of controlled evaluation studies", British Medical Journal, 324(7330), 2002, pp. 153157. 